\title{
Soft X-ray absorption spectroscopy investigation of the surface chemistry and treatments of copper indium gallium diselenide (CIGS)
}

\section{Author List}

Craig Schwartz ${ }^{1,2}$, Dennis Nordlund ${ }^{1}$, Dimosthenis Sokaras ${ }^{1}$, Miguel Contreras ${ }^{3}$, Tsu-Chien Weng ${ }^{1,4}$, Lorelle Mansfield ${ }^{3}$, Katherine E. Hurst ${ }^{3}$, Arrelaine Dameron ${ }^{3}$, Kannan Ramanathan $^{3}$, David Prendergast ${ }^{2}$, Steven T. Christensen ${ }^{3 *}$

${ }^{1}$ Stanford Synchrotron Radiation Lightsource, SLAC National Accelerator Laboratory, 2575 Sand Hill Rd, Menlo Park, 94025 CA, USA

2The Molecular Foundry, Lawrence Berkeley National Laboratory, 1 Cyclotron Road, Berkeley, CA 94720

${ }^{3}$ National Renewable Energy Laboratory, 15013 Denver West Parkway, Golden, 80401 CO, USA

${ }^{4}$ Center for High Pressure Science \& Technology Advanced Research, \#6-408, 1690 Cailun Rd., Pudong, Shanghai 201203, P. R. China

${ }^{*}$ Corresponding author.

\begin{abstract}
The surface and near surface structure of copper-indium-gallium-selenide (CIGS) absorber layers is integral to the producing a high-quality photovoltaic junction. By using X-ray absorption spectroscopy (XAS) and monitoring multiple elemental absorption edges with both theory and experiment, we are able to identify several features of the surface of CIGS as a function of composition and surface treatments. The XAS data shows trends in the near surface region of oxygen, copper, indium and gallium species as the copper content is varied in the films. The oxygen surface species are also monitored through a series of experiments that systematically investigates the effects of water and various solutions of: ammonium hydroxide, cadmium sulfate, and thiourea. These being components of cadmium sulfide chemical bath deposition (CBD). Characteristics of the CBD are correlated with a restorative effect that produces as normalized, uniform surface chemistry as measured by XAS. This surface chemistry is found in CIGS solar cells with excellent power conversion efficiency $(<19 \%)$. The results provide new insight for CIGS processing strategies that seek to replace CBD and/or cadmium sulfide.
\end{abstract}

\section{Key words}

Copper-indium-gallium-selenide, CIGS, XAS, chemical bath deposition, surface analysis

\section{Introduction}

Page 1 of 19 
Copper-indium-gallium-selenide (CIGS) solar cells have been in development for over 20 years, and have shown efficiencies of over 22\%.[1]Polycrystalline thin film technologies offer the promise of reduced processing costs while still providing high power conversion efficiencies (PCEs)[2]. Several strategies have been developed to fabricate the multi-element compound including evaporation, sputtering, metal selenization, and solution deposition, to list a few. A major theme from research in CIGS has revolved around the surface and near surface chemistry of CIGS. The topic has been reviewed several times.[3-5] It suffices to say that the multi-element CIGS surface chemistry is very complex and yet the formation of high-quality photovoltaic junctions is evident in device performance.[1]

The CIGS architecture commonly employs chemical bath deposition (CBD) of cadmium sulfide to fabricate the interfacial layer for the front contact. The combination of CBD and CdS appears to be the best method to produce high performing CIGS.[6, 7] CdS has good energy-band alignment with the CIGS to minimize transport losses. The overall field has been reviewed in detail by both Todorov and Huang.[8, 9] CdS however, limits the potential photo current due to its $2.4 \mathrm{eV}$ band gap and presents toxicity concerns. [10] By some estimates the additional photocurrent that could be gained could improve overall performance $10 \%$ making $>23 \%$ PCE devices. Nevertheless, the problem is elusive and has not fully been resolved. Our approach to this challenge is to investigate CIGS surface and related CBD surface treatments with element specific chemical analysis in the form of X-ray absorption spectroscopy to deconvolute the role of the CBD processing and CdS material. We recently reported on CdS films as part of this effort and herein focus our efforts on the CBD processing.[11] This methodology can be applied to materials and processes being considered for replacing $\mathrm{CdS}$ such as $\mathrm{ZnO}_{\mathrm{x}} \mathrm{S}_{1-\mathrm{x} \cdot[}[12,13]$

By probing the CIGS surface with element specific chemical analysis before and after chemical bath processing, we gain new insight into the surface chemistry of CIGS. This is motivated by an important question: how does the CBD of CdS react to the surface chemistry produced by CIGS absorber processing? To answer this, we investigate the CIGS surface with soft X-ray absorption spectroscopy from three perspectives: 1) CIGS bulk composition effect on the surface; 2 ) a stepwise evaluation of the CBD process; 3 ) chemical bath treatment of CIGS with deliberate surface degradation. The metal ratio of the CIGS bulk film will determine in part the chemical termination at the surface and will play a role in the formation of the interface with CdS. Understanding the chemical species at the surface gives insight on how the chemical bath processing develops the surface chemistry. The second component to this study demarks the possible effects from each constituent in the chemical bath processing. CBD requires the CIGS absorbers to be exposed to ambient conditions following deposition and the third experiment investigates this in the extreme.

We use XAS, an element-specific probe that measures the electronic and atomic structure of a material following excitation from a core state into an unoccupied state.[14] As part of this, an unlikely player is investigated to understand the surface chemistry: oxygen. Probing how oxygen bonds to the CIGS surface gives insight into reactive species that likely play a role in the formation of the interface. The richest information was obtained from the near surface region $x<50 \mathrm{~nm}$ by measuring total electron yield (TEY) of the excited core electron decay process. The understanding gained from XAS can be increased by comparing experimental spectra to those calculated of model systems by 
density functional theory (DFT).[15] This DFT methodology is specific to XAS that takes model structures as input. With this in mind, we can investigate the surface and nearsurface composition with respect to the elemental make-up related to CIGS. The measurements included $\mathrm{Cu}, \mathrm{Ga}$, and $\mathrm{O}$. These elements are well situated to be studied based on the X-ray absorption cross-section, their presence in CIGS devices, and the experimental capabilities of the synchrotron end station. Oxygen can be thought of as a 'stop bath', equivalent to what is used to halt the developer used in making print photographs and showing the reactive species, particularly at the surface. XAS of oxygen is well understood with extensive literature and in terms of XAS computation. Furthermore, oxygen interaction with CIGS has not always been emphasized in investigating the processing of CIGS.

The XAS was measured on many different CIGS samples after deposition and selected chemical bath processing. The CBD of CdS is performed in basic solution $\left(\mathrm{NH}_{4} \mathrm{OH}\right)$ using both cadmium sulfate $\left(\mathrm{CdSO}_{4}\right)$ and thiourea $\left(\mathrm{SC}\left(\mathrm{NH}_{2}\right)_{2}\right)$ as precursors at $\sim 65^{\circ} \mathrm{C}$. [16] Complete formation of CdS film from CBD was avoided to maintain a surface sensitivity for the XAS signal. The CBD process was thus studied piecewise where CIGS absorbers were exposed to different steps in of the CBD process with subsequent XAS measurements.[1721] The approach is derived from experiments in CIGS research where it was found that a 'partial electrolyte' solution of $\mathrm{NH}_{4} \mathrm{OH}$ and $\mathrm{CdSO}_{4}$ correlates with improved device performance, albeit not as significant as with complete CBD of CdS.[22] This is referred to hereafter as the partial electrolyte dip (PED).

\section{Materials and Methods}

CIGS was coevaporated on molybdenum-coated soda-lime glass using the threestage method developed at the National Renewable Energy Laboratory.[23] All device architectures consisted of the following: soda lime glass substrate, molybdenum, CIGS, CdS window layer, zinc oxide window layer, aluminum-doped zinc oxide, metal contacts. Two different deposition systems were employed in this study. Data will be designated as either a C-system or P-system to identify the source material. Both the C- and P-systems use coevaporation that is optimized for the instrument to produce the best possible device results. We include results from both the C- and P-systems to assess similarities in CIGS absorbers despite the source deposition system. Samples were stored in a nitrogen environment following deposition and during transport. Handling procedures were conducted such that exposure to air was minimized. As discussed above, this work emphasizes three experiments designed to investigate the processing of CIGS surface: 1 ) CIGS bulk composition effect on the surface; 2) a stepwise evaluation of the CBD process; 3 ) chemical bath treatment of CIGS with deliberate surface degradation. The following summarizes the experiments performed to produce the samples.

Devices were fabricated[23] from absorber films sectioned from the same sample that received the synchrotron measurements. Devices consisted of a CdS layer by CBD, intrinsic zinc oxide, aluminum doped zinc oxide, and contact metallization.

Composition effects. The standard coevaporation process was modified to change the ratio of metal evaporation to produce $\mathrm{Cu} / \mathrm{In}+\mathrm{Ga}$ ratios of: $0.93,0.86,0.84,0.79,0.76$, and 0.71 . These samples were grown in the P-system. 
Stepwise chemical bath treatments. Five pieces of CIGS were individually submersed for 13 minutes at $65^{\circ} \mathrm{C}$ in solutions of the following: 1) deionized water; 2) $\mathrm{NH}_{4} \mathrm{OH}(1 \mathrm{mM})$; 3) Thiourea/ $\mathrm{NH}_{4} \mathrm{OH}(0.1 \mathrm{mM} / 1 \mathrm{mM})$; 4) $\mathrm{CdSO}_{4}(2 \mu \mathrm{M})$; 5) $\mathrm{CdSO}_{4} / \mathrm{NH}_{4} \mathrm{OH}(2 \mu \mathrm{M} / 1 \mathrm{mM})$. The $\mathrm{CdSO}_{4} / \mathrm{NH}_{4} \mathrm{OH}$ treatment is hereafter referred to as the cadmium partial electrolyte dip or PED. The CIGS films was grown in the $C$-system.

Surface degradation. The surface degradation was accomplished where sections of CIGS were exposed to oxygen plasma or argon sputtering. Remote oxygen plasma exposures were performed in a plasma CVD system (NPE-4000, Nano-Master Inc.) with conditions: 1 minute exposure, 0.7 Torr, $75 / 5 \mathrm{sccm}$ of $\mathrm{O}_{2} / \mathrm{N}_{2}, 500 \mathrm{~W}$, and $+50 \mathrm{~V}$ DC sample bias. Argon sputtering was performed in a custom vacuum at a base pressure for the experiment of $4 \times 10^{-6}$ Torr and operating pressure with argon at $7 \times 10^{-4}$. The sputtering gun was set to a $55 \mathrm{~V}$ discharge at $30 \mathrm{~mA}$ for 1 minute. Each case included two samples where one received a chemical bath treatment in addition to the oxygen plasma or argon sputtering. Witness samples of as-deposited CIGS and CBD-treated CIGS were also included. The CIGS films were grown in the C-system.

XAS Measurements. Measurements were taken at end station 10-1 at Stanford Synchrotron Radiation Laboratory and aligned to known standards. Nominal resolution was $\triangle \mathrm{E} / \mathrm{E}$ of $\sim 2 \times 10^{-4}$. The spectra were collected by both total electron yield (TEY) by monitoring the drain current generated by the incident photons, as well as by monitoring total fluorescence yield using a photodiode. Auger electron yield (AEY) measurements were obtained by using a cylindrical mirror analyzer and only collecting the auger electrons from the relevant element.

Calculated XAS Spectra - XAS spectra were calculated using a method that has been detailed previously.[24] We employ QUANTUM ESPRESSO within the plane-wave pseudopotential orbital-occupancy constrained DFT employing the PBE functional.[25] The transition amplitudes are calculated according to Fermi's golden rule for X-ray absorption cross-sections:

$$
\sigma(\omega)=4 \pi^{2} \alpha_{0} \hbar \omega \sum_{f}\left|M_{i \rightarrow f}\right|^{2} \delta\left(E_{f}-E_{i}-\hbar \omega\right) .
$$

In this case, $\alpha_{0}$ is the fine structure constant, $\hbar \omega$ is the energy of the absorbed photon and $M_{i \rightarrow f}$ are the transition amplitudes between the initial $(i)$ and final $(f)$ states, with a corresponding energy difference of $E_{f}-E_{i}$. The initial state is always the $1 s$ orbital of the excited atom and the final states are the unoccupied Kohn-Sham eigenstates derived from a self-consistent field computer within the excited electron and core hole (XCH) approximation for the electronic final state.[15]

The excited-state electron density is computed self consistently with the replacement of the ground-state pseudopotential of the core-excited atom in the system with that derived from a similarly core-excited isolated atom (for the $\mathrm{O} \mathrm{K}$-edge, we assume the configuration of $1 s^{1} 2 s^{2} 2 p^{5}$ and $1 s^{2} 2 s^{2} 2 p^{5} 3 s^{2} 3 d^{10} 3 p^{2}$ ) to model the hole and the addition of one extra (excited) electron to the total number of ground state valence electrons to this system.[15]

The sum over final states includes both electronic band indices and wave vectors. In this work, this required multiple $k$-points, and we lower the computational cost by exploiting a scheme from which the entire Brillouin zone can be generated based only from the zone center. 
Spectra are aligned relative to a theoretical standard - the isolated excited and ground state atom under the same periodic boundary conditions. This is identical to computing a difference in formation energies between the excited and ground states, and formation energies are numerically well defined within a pseudopotential framework.[24] In practice, we aligned the computed spectrum to an experimentally well-defined system $\left(\mathrm{H}_{2} \mathrm{O}\right.$ and $\left.\mathrm{GaPO}_{4}\right)$ and use the same constant-of-alignment in all further calculations using the same excited state pseudopotential. The spectra are further broadened $20 \%$ to account for the well-known tendency of DFT to underestimate the band gap.[26] The density of states was calculated within QUANTUM ESPRESSO.[27] Calculated spectra were convoluted with Gaussian functions of $0.3 \mathrm{eV}$ (oxygen) and $0.6 \mathrm{eV}$ (gallium) FWHM.

Structures. The structure of all the pure crystals here were taken from the literature.[28] When a defect was added or removed, the target atom was swapped with the appropriate atom or defect and the structure was then relaxed within QUANTUM ESPRESSO. Forces were minimized to within $10^{-6}$ Rydberg. These are not intended to be definitive structures but rather examples of the types of defects that might be present.

\section{Results}

Figure 1 shows the experimental and calculated oxygen $1 s$ spectra for a series of conditions. The experimental data were taken on a series of $P$-system CIGS surfaces with the composition ratio, $X=\mathrm{Cu} /(\mathrm{In}+\mathrm{Ga})=0.93,0.86,0.84,0.79,0.76$, and 0.71 . The calculated spectra were developed to interpret the effects of composition. The measurements constitute a sampling of the near-surface region. The three dashed lines correspond to the $1 s->2 p$ states of oxygen for double-bonded oxygen $-R=0(532 \mathrm{eV})[14,29-31], \mathrm{Cu}_{2} \mathrm{O}(533$ $\mathrm{eV})[32]$, and hydroxyl $-\mathrm{OH}(534 \mathrm{eV})[14,29,33]$. The calculated spectra were derived from structures obtained in the literature including: $\mathrm{SeO}_{2}, \mathrm{Cu}_{2} \mathrm{O}$,[34] liquid water,[35] $\alpha$ $\mathrm{Ga}_{2} \mathrm{O}_{3}$,[36] $\mathrm{CuO}$,[34] and $\mathrm{In}_{2} \mathrm{O}_{3}$ [37]. Other species that were ruled out due to poor agreement included condensed $\mathrm{O}_{2}$ [38] and $\mathrm{CdO}$ [37]. The peak at $532 \mathrm{eV}$ assigned to $-R=0$ in the experimental spectra remains roughly constant. The peak assigned to $\mathrm{Cu}_{2} \mathrm{O}$ generally increases with increasing $\mathrm{Cu}$ content. The $-\mathrm{OH}$ peak also appears to remain constant, but is likely convoluted with other contributions from the surface.

Figure 2 shows the $P$-system device performance for samples as function of copper composition, $X$, including: open circuit voltage $\left(V_{O C}\right)$, short circuit current density $\left(U_{S C}\right)$, fill factor $(F F)$, and power conversion efficiency (PCE). Trends for the J-V parameters are due to $\mathrm{Cu}$ content affecting, among others, the optical (bandgap) and electrical properties (carrier concentration) of the CIGS materials. Best performance peaks near 0.83-0.84 $X$ where carrier concentrations generally reach mid $10^{16} \mathrm{~cm}^{-3}$ values resulting in the highest $V_{o c}$ and $F F$ values. Higher $\mathrm{Cu}$ contents lead to conductivities that are too high (non optimum) leading to lower $J_{s c}$ values (due to a reduced space charge region and thus a loss in collection of IR photons) and lower $V_{o c}$ values as a consequence

Figure 3 shows a series of oxygen $1 s$ XAS spectra of $C$-system CIGS absorbers taken by both total electron yield and auger electron yield (AEY). The TEY probes a depth on the order of 10-50 nm, whereas AEY is more surface-sensitive, probing only 1-2 nm. Both signals are collected simultaneously and are presented to investigate the possibility of differences in the surface versus the near-surface region. Spectra are shown for a variety of CIGS after-processing treatments: as grown, hot water, thiourea/ $\mathrm{NH}_{4} \mathrm{OH}, \mathrm{NH}_{4} \mathrm{OH}, \mathrm{PED}$, and 
a film that produced over 19\% PCE. The peak at $530.5 \mathrm{eV}$ is found in the more bulksensitive TEY, but not in the more surface-sensitive AEY. This peak is the only feature that shows a difference in the spectral-line shape for the AEY and TEY. It is not present in $P$ system spectra but is thought to be benign as it occurs even the $19 \%$ device. The same peak found previously at $532.2 \mathrm{eV}$ on CIGS is once again found here. The inset shows the height $(\Delta y)$ of the peak at $532.2 \mathrm{eV}$ for the measurements determined graphically. The PED sample is the clear standout but also the high-PCE sample is also very strong with no additional treatments. The as-grown sample and $\mathrm{NH}_{4} \mathrm{OH}$-treated samples appear very similar. Comparing them to the hot water treatment shows additional intensity in the 532$535 \mathrm{eV}$ region.

Figure 4 shows the XAS of $C$-system absorbers for the $01 s, \mathrm{Cu} 2 p$, and $\mathrm{Ga} 2 p$ edges for sample treatments that include as grown, oxygen plasma, and argon implantation both as treated and after the PED. The changes in the spectral features are highlighted by shaded regions. The surface treatments lead to distinct changes in the XAS that are significantly altered by the PED. The 01 s XAS show the formation of the $532 \mathrm{eV}$ resonance associated with $-R=0$ and a trend to the similar spectral shape. The $\mathrm{Cu} 2 p$ data show the formation of a shoulder near $927 \mathrm{eV}$ and somewhat smeared spectral features for the Arimplanted case. Other spectra are not significantly different and the PED appears to promote a consistent spectral shape in all cases. The Ga $2 p$ data continue to show the effects that the PED promotes a consistent spectral shape. The samples not treated with PED show Ga $2 p$ spectra that have similarities for the as grown, oxygen plasma, and argon implanted samples.

The $C$-system device performance for samples prepared with the treatments in Figure 4 is presented in Figure 5 . The $J$ - $V$ data in Figure 5 (a)-(c) are taken from the best performing sample in each treatment. The best device performance gave a $16.4 \% P C E$. Devices were processed with a CBD CdS layer and standard window and contact layers. In Figure $5(\mathrm{~d})-(\mathrm{g})$, the $V_{O C}, J_{S C}, F F$, and $P C E$ show trends of decreasing performance from the as grown, oxygen plasma, and argon implanted cases.

Figure 6 shows the results of calculations at the oxygen $1 s$, copper $2 p$, and gallium $2 p$ edges and some example experimental spectra for comparison. The $01 s$ spectra include: the point defect case where oxygen replaces selenium in CIGS; $\mathrm{SeO}_{2}, \mathrm{CdSO}_{4} ; \operatorname{In}_{2} \mathrm{O}_{3}$; $\alpha-\mathrm{Ga}_{2} \mathrm{O}_{3}$. The calculated CIGS $\mathrm{Cu} 2 p$ spectra are shown in Figure 6 (b). Figure 6 (c) gives Ga 2p spectra. The previously highlighted lines of $530.5 \mathrm{eV}, 532.2 \mathrm{eV}$, and $533.2 \mathrm{eV}$ are indicated for comparison. Calculated spectra of the Ga $2 p$ are shown for CIGS (green), GaS (orange), $\mathrm{Ga}_{2} \mathrm{~S}_{3}$ (light blue), $\alpha-\mathrm{Ga}_{2} \mathrm{Se}_{3}$ (dark blue), $\beta-\mathrm{Ga}_{2} \mathrm{Se}_{3}$ (yellow), GaSe(purple), $\alpha-\mathrm{GaO}$ (red), $\beta$-GaO (black). The main features following PED in the experiment are marked at 1120.5 and $1122.1 \mathrm{eV}$. From this, the Ga $2 p$ calculations support an oxidized Ga species for the as grown and oxygen plasma treated samples whereas the PED promotes spectral features with gallium in a CIGS and/or $\mathrm{Ga}_{2} \mathrm{Se}_{3}$ environment.

\section{Discussion}

Using the 0 1s XAS as a metric of the surface composition, we can see that the CIGS surface exhibits a significant variety of species with respect to fabrication and processing. Nominally identical materials can be quite different as seen in the difference between the 
as grown spectra in Figures 1, 3, and 4. While this work is not comprehensive with respect to all CIGS deposition and processing, the intent is to provide a diverse dataset from actual devices for a perspective that will better inform device makers. Oxygen is not designed to be present at all; it is nevertheless a consequence of many CIGS processing strategies as exposure to air often occurs in handling following deposition. The data in Figure 1 are a starting point to appreciate what oxygen species may be present with respect to film composition. The direct connection to composition is in the systematic increase of the peak shoulder at $533 \mathrm{eV}$ assigned to $\mathrm{Cu}$ (I) oxygen species similar to $\mathrm{Cu}_{2} \mathrm{O}$. The data does not represent a true depth profile, however the copper content in the near surface region may be compared.

The partial electrolyte treatments shown in Figure 3 arise from work investigating the chemical bath deposition (CBD) of cadmium sulfide. Ramanathan et al. showed that reasonable device performance could result by the PED after standard window and contact device layers were added.[39] This motivates the present experiment to dissect the PED for clues about specific components of the process. The spectral features of primary interest consist of the peak at $532.2 \mathrm{eV}$ and $\sim 534-35 \mathrm{eV}$ region.

While the best XAS results accompany the full PED, the least influential surface treatment relates to ammonium hydroxide solution treatment only. The basic environment of this treatment would likely result in the removal of surface oxide material. The result nevertheless appears to produce a surface chemistry similar to hot water and as grown conditions. The other solution treatments (PED, thiourea $+\mathrm{NH}_{4} \mathrm{OH}, \mathrm{CdSO}_{4}$ ) appear to chemically alter the surface composition to produce the $-R=0$ resonance at $532 \mathrm{eV}$. The basic environment of this treatment would likely result in the removal of surface oxide material. The case of PED and $\mathrm{CdSO}_{4}$ suggest that $\mathrm{CdSO}_{4}$ reacts with the CIGS surface due to the presence of cadmium sulfate. That the PED could promote a favorable surface[39] chemistry as measured by XAS is a key insight gained from this study.

The positive effects of the PED are further illustrated in Figure 4. These data show that the PED can be used to restore the surface termination following oxidation and damage. The initial CIGS oxygen, copper, and gallium spectra whether bare or treated with an oxygen plasma evolves to a similar spectrum. The $01 s$ data show a consistent peak at $532.2 \mathrm{eV}(-\mathrm{Se}=0)$ after PED although in the Ar implant case additional damage was not completely removed. The removal of a peak at $536 \mathrm{eV}$ which could be related to oxidized indium (c.f. Fig 3) is also observed.

The $\mathrm{Cu} 2 p$ XAS in various conditions is shown in Figure 4 (b) by total electron yield. For the oxygen plasma treated and as grown samples, the differences between the dip and no PED are not significant. This is likely because $\mathrm{Cu}$ is only found in the CIGS layer and the oxygen plasma does not cause significant damage. Thus, the PED does not change the copper, particularly when there is no damage to it. The Ar implanted shows a peak in the pre-edge region $(\sim 925.5 \mathrm{eV})$. This shoulder is related to $\mathrm{Cu}$ moving into a higher oxidation state, $\mathrm{Cu}(\mathrm{II})$, as compared with the $\mathrm{Cu}$ in CIGS $\mathrm{Cu}$ (I). This peak is consistent with previous results on copper.[40] Argon implantation has been observed to introduce significant damage to materials that are susceptible to oxidation.[41] The PE treatment appears to restore the copper to its previous oxidation state. The restoration occurs for both the TEY and fluorescence yield (not shown), implying the PED infiltrates the material as far as the Argon is implanted and thus largely restores the near surface structure. 
The Ga $2 p$ XAS collected by total electron yield in the same conditions as oxygen and copper is shown in Figure 4 (c) and generally consists of 2 peaks. The bare CIGS gallium is largely unchanged by treating the surface with an oxygen plasma with two peaks at 1120.3 $\mathrm{eV}$ and $\sim 1121.7 \mathrm{eV}$. The argon implantation does appear change the gallium spectrum to a single broad peak, however all of these spectra are transformed to nearly identical spectra independent of the damage with peaks located at 1120.5 and $1122.1 \mathrm{eV}$ following PED.

The device results shown in Figure 5 for the PED treatment indirectly support the potential impact the CBD has on the CdS-CIGS interface and cell performance. Devices with a best-case performance at $16.4 \%$ PCE show decreasing performance for the oxygen plasma treated and argon implanted cases. Nevertheless, the deleterious treatments to the CIGS surface prior to device fabrication did not completely terminate performance. It is somewhat surprising that some of argon-implanted devices still operate. All of these samples had a standard CBD CdS processing treatment. This provides experimental evidence that the PED and CBD processing are linked in their effects on CIGS devices and it is reasonable to be concluded that the CBD process produces an interface where the chemical environments of the $\mathrm{Cu}, \mathrm{In}, \mathrm{Ga}$, and Se are similar to that observed with XAS. This is particularly valuable as the CdS-CIGS interface (or any interface for that matter) is extremely difficult to isolate and characterize.

The restorative effect is crucial support that the CBD (or PED) processing produces a high quality CdS-CIGS interface via chemical reaction. The results from Figure 3 support that the high-efficiency cell and the PED have similar surface chemistries. Nevertheless, it could be argued that elements of the surface chemistry is present on all CIGS and that the chemical bath processing normalizes many different surface chemistries to the that which produces the XAS results for the PED and high efficiency devices. The observed effects hold for the near surface region $(x<50 \mathrm{~nm})$ of the CIGS surface based on the TEY data collection of the XAS. Furthermore, it is reasonable that the CBD (PED) processing effects result for both the indium and selenium in CIGS despite the XAS for these elements not being experimentally tractable for this study. The type of chemical reaction the CBD (PED) processing involves both removal of oxygen species by the base conditions and additional chemistry from species like $\mathrm{Cd}^{2+}$. Indeed the $\mathrm{Cd}^{2+}$ would coordinate to selenium surface atoms in solution. When the solution is removed the selenium could compensate by scavenging oxygen to produce the observed $-\mathrm{Se}=0$ termination. In a completed CBD of CdS the coordinated -Se-Cd- bonding would be present and would constitute the makings of a high-quality interface.

The focus of this study has been limited to the effects of solution processing CdS which result in subtle differences in the surface chemistry that appear to have some impact on device processing. The effort attempted to include as many as factors that may be tied to the performance, such as CIGS composition and exaggerating the effects of oxidation. The goal of this was to provide device makers with as broad as knowledge possible to account for alternative methods for both CIGS absorber and CdS processing. The results presented here provide a deeper understanding of a CBD/PED strategy that nevertheless can inform other methods that use different solution precursors, vapor phase deposition, or sputtering.

\section{Conclusions}


The CIGS surface and interface with CdS in solar cells is challenging to adequately characterize because of number of potential chemical species. This work has investigated the chemical structure of CIGS as a function of deposition source, film composition, and surface treatments to inform CIGS solar cell fabrication. Based on the experimental and theoretical XAS, the following can be expected: 1) oxygen is present in the near-surface region $(x<50 \mathrm{~nm}) ; 2)$ the bulk composition strongly influences the surface composition; 2) the $\mathrm{Cu}$, In, $\mathrm{Ga}$, Se species are chemically restored to an environment consistent with the CIGS crystallographic structure; 3 ) $-\mathrm{Se}=0$ chemical termination results from the chemical processing of the PED; 4) any surface oxidation or contamination is mitigated through the CBD (PED) process. This is new information on how high-quality interfaces at critical materials junctions form in CIGS solar cells. These results can guide development of processing strategies that do not incorporate cadmium-based wet chemical treatments. For example: does the CBD of zinc-oxygen-sulfur buffer layers produce a similar surface chemistry? Or can vapor-based strategies achieve this surface chemistry? Answering these questions will help validate new strategies to develop better performing and cadmium free CIGS solar cells.

\section{Acknowledgements}

The Stanford Synchrotron Radiation Lightsource and Molecular Foundry are National User Facilities operated by Stanford University and the University of California Berkeley for the U.S. Department of Energy, grants DE-AC02-76SF00515 and DE-AC0205CH11231 respectively. The Molecular Foundry portion of this work was performed under a user proposal. The work was supported by the U.S. Department of Energy under Contract No. DE-AC36-08-G028308 with the National Renewable Energy Laboratory. The project was supported by U.S. Department of Energy, Office of Energy Efficiency \& Renewable Energy, Solar Energy Technology Office BRIDGE program. We would like to thank Erik Nelson and Matthew Lattimer for their excellent beamline support. 
Figures

Figure 1

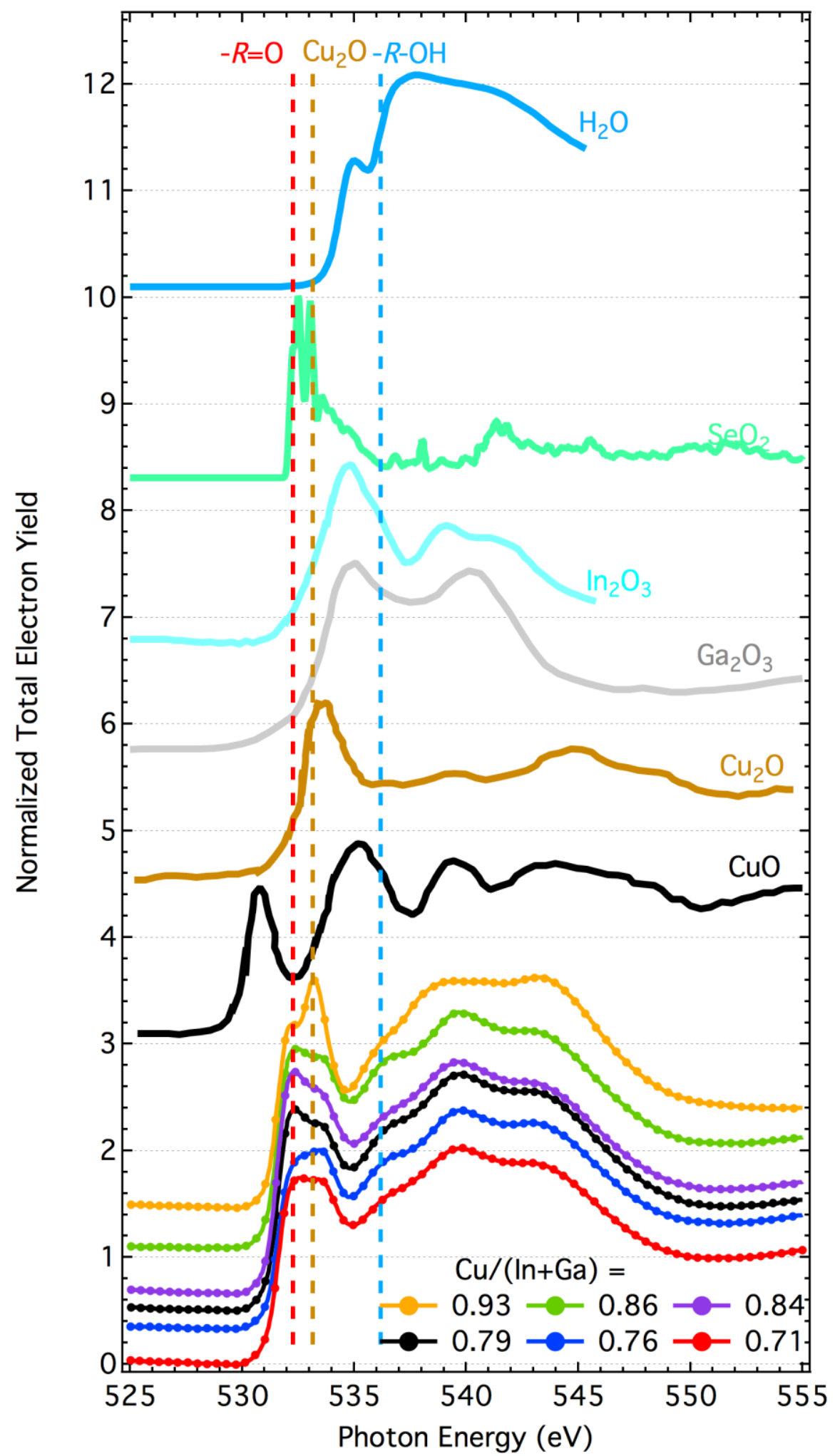

Page 10 of 19 
Figure 1. Experimental and calculated oxygen $1 s \mathrm{X}$-ray absorption spectra for CIGS and related oxides. The CIGS data from absorbers grown in the NREL $P$-system represent different metal composition given by the $\mathrm{Cu} /(\mathrm{In}+\mathrm{Ga})$ ratio: $0.93,0.86,0.84,0.79,0.76$, and 0.71. Calculated spectra for possible oxides that could be present included $\mathrm{CuO}, \mathrm{Cu}_{2} \mathrm{O}$, $\mathrm{Ga}_{2} \mathrm{O}_{3}, \mathrm{In}_{2} \mathrm{O}_{3}$, and $\mathrm{H}_{2} \mathrm{O}$. The CIGS show evidence of $\mathrm{SeO}_{2}, \mathrm{Cu}_{2} \mathrm{O}$ and $\mathrm{H}_{2} \mathrm{O}$ as components of the surface/near-surface composition. Other species are also present but less distinguishable. The CIGS composition shows a trend where the $\mathrm{Cu}_{2} \mathrm{O}$ contribution decreases with decreasing $\mathrm{Cu}$ content. 


\section{Figure 2}

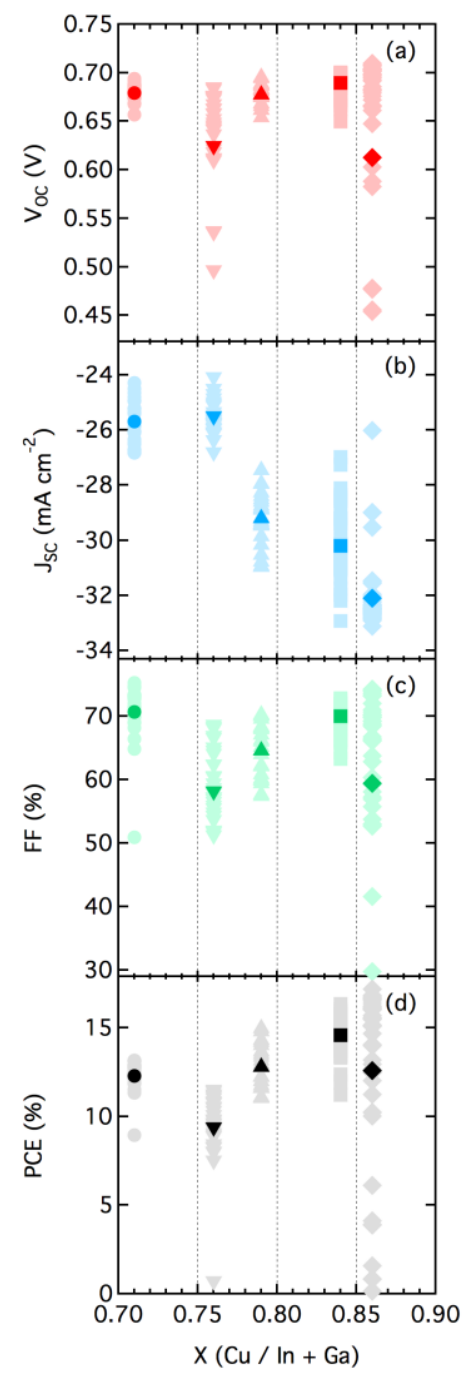

Figure 2. Device performance as function of copper composition, $X$ : a) open circuit voltage $\left(V_{O C}\right)$; b) short circuit current density $\left(J_{s c}\right)$; c) fill factor $(F F)$; d) power conversion efficiency $(P C E)$. Devices were fabricated as companion samples to those presented in Figure 1. The $F F$ depends most on the copper content as expected. The trend $J_{S C}$ is related to a changing band gap due to copper composition. 


\section{Figure 3}

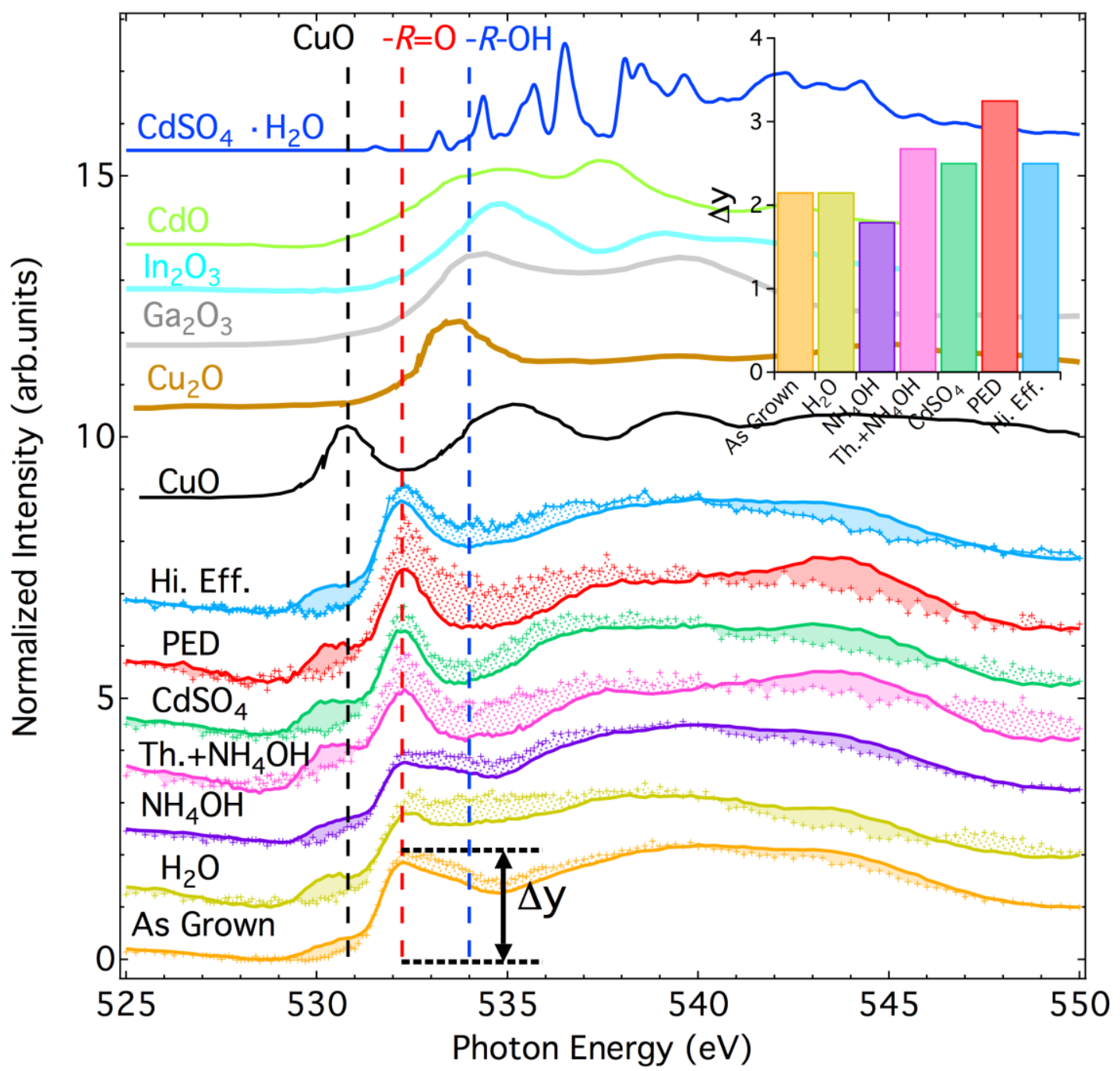

Figure 3. Experimental and calculated $\mathrm{x}$-ray absorption spectra of the oxygen $1 s$ of CIGS surfaces having undergone various treatments, with total electron yield as a solid line and the more surface-sensitive Auger electron yield as crosses. The data were collected on absorbers grown in the NREL $C$-system. The series of aqueous treatments are as grown (orange), hot water (yellow), ammonia (purple), thiourea and ammonia (pink), $\mathrm{CdSO}_{4}$ (green), the PED (red) and finally in an as grown CIGS film with PCE > 19\% cell (blue). The peak at $532.2 \mathrm{eV}$ is present always, but the peak at $530.5 \mathrm{eV}$ is only present in the more bulk sensitive TEY measurements. Some amount of $\mathrm{CuO}$ was present in these films while the metal oxides are not prominent. The $-R=0$ feature, most likely due to $-\mathrm{Se}=0$, is a prominent feature for PED and high-performance materials. 


\section{Figure 4}

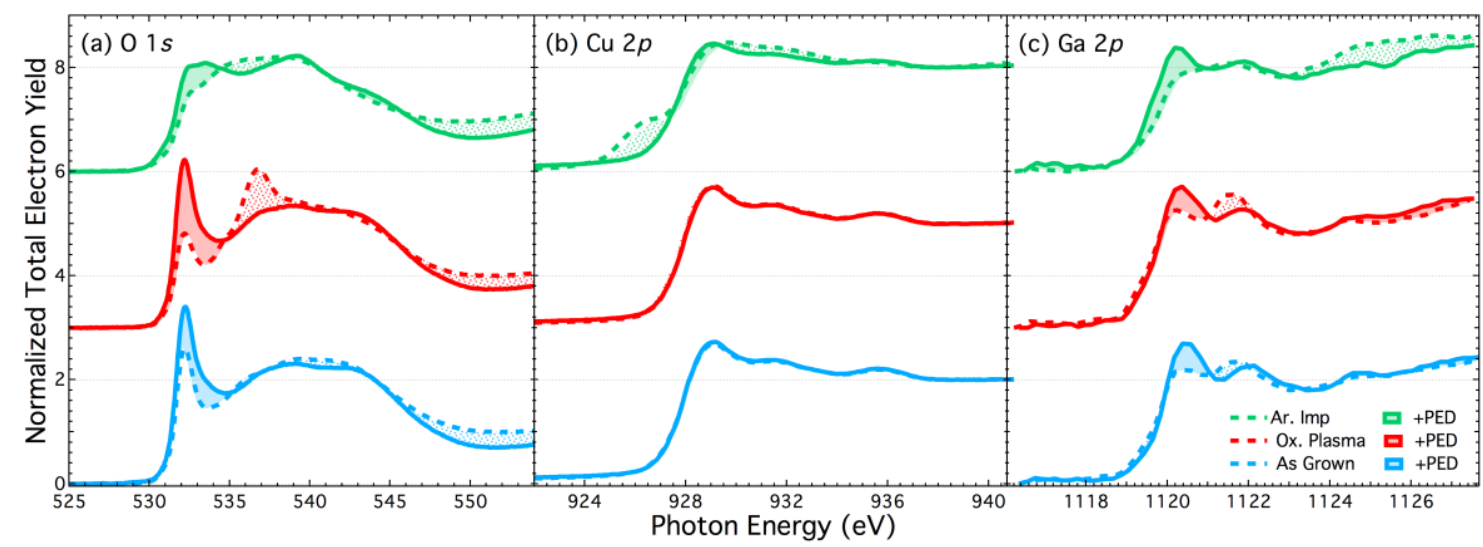

Figure 4. Experimental X-ray absorption spectra of NREL $C$-system CIGS absorbers of the: (a) $01 s$, (b) $\mathrm{Cu} 2 p$, and (c) Ga $2 p$ collected by total electron yield. Dashed lines represent measurements prior to the partial electrolyte dip (PED) for the as grown, oxygen plasma, and argon implanted sample conditions. The solid lines represent the measurements for the respective sample conditions after PED. The shaded region illustrates to the differences due to the PED. All three-absorption edges show evidence that the PED produces a consistent XAS signature despite sample treatments. 
Figure 5

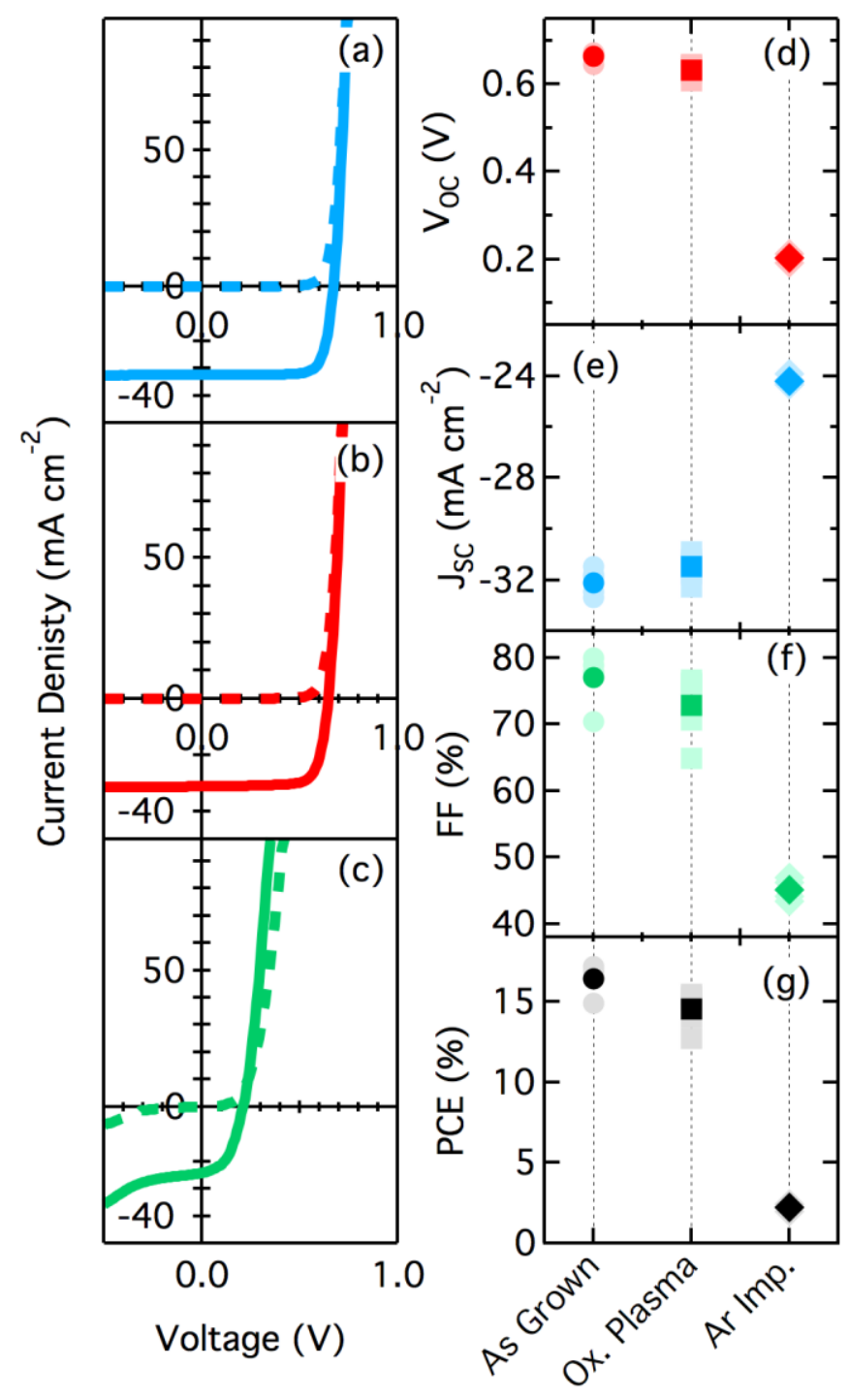

Figure 5. Device performance for the surface modified CIGS films from Figure 4. Surface treatments included oxygen plasma or argon implantation followed by CBD CdS.

Performance drops from the control, as grown, sample to oxygen plasma and then argon implantation. Despite this, devices are still operational in all cases suggesting that the CBD process, like the PED, promotes the needed surface/interfacial chemistry for a functional device. 


\section{Figure 6}

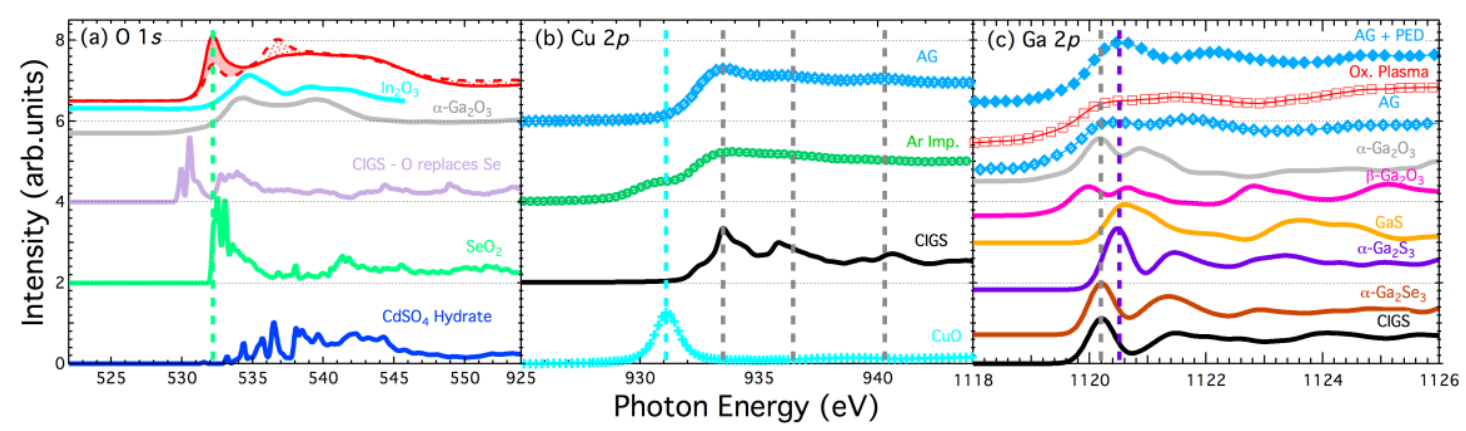

Figure 6. Calculated (solid lines) and experimental (combined markers and lines) spectra for the $01 s(\mathrm{a}), \mathrm{Cu} 2 p(\mathrm{~b})$, and $\mathrm{Ga} 2 p$ (c) edges. Comparison of example experimental spectra of CIGS helps: eliminate point defects ( 0 replaces Se) and contamination $\left(\mathrm{CdSO}_{4}\right)$; validate calculations ( $c f$. $\mathrm{Cu} 2 p$ experimental to calculated); establish the role in of the PED in replacing highly oxidized species to a environment consistent with the CIGS structure. For example, the Ga $2 p$ data shows that an as-grown surface contains oxidized Ga similar to $\mathrm{Ga}_{2} \mathrm{O}_{3}$ that is converted to something more consistent with a CIGS environment. 


\section{References}

[1] S. Frontier, Solar Frontier Achieves World Record Thin-Film Solar Cell Efficiency: $22.3 \%$, in, 2015.

[2] M. Dhankhar, O.P. Singh, V.N. Singh, Physical principles of losses in thin film solar cells and efficiency enhancement methods, Renew. Sust. Energ. Rev., 40 (2014) 214-223.

[3] D. Hariskos, S. Spiering, M. Powalla, Buffer layers in $\mathrm{Cu}(\mathrm{In}, \mathrm{Ga}) \mathrm{Se}_{2}$ solar cells and modules, Thin Solid Films, 480 (2005) 99-109.

[4] N. Naghavi, D. Abou-Ras, N. Allsop, N. Barreau, S. Bucheler, A. Ennaoui, C.H. Fischer, C. Guillen, D. Hariskos, J. Herrero, R. Klenk, K. Kushiya, D. Lincot, R. Menner, T. Nakada, C. Platzer-Bjorkman, S. Spiering, A.N. Tiwari, T. Torndahl, Buffer layers and transparent conducting oxides for chalcopyrite $\mathrm{Cu}(\mathrm{In}, \mathrm{Ga})(\mathrm{S}, \mathrm{Se})_{2}$ based thin film photovoltaics: present status and current developments, Progress in Photovoltaics, 18 (2010) 411-433.

[5] S. Siebentritt, Alternative buffers for chalcopyrite solar cells, Solar Energy, 77 (2004) 767-775.

[6] C.W. Chen, H.W. Tsai, T.T. Wu, Y.T. Yen, Y.C. Wang, C.H. Hsu, W.C. Tsai, H.S. Tsai, C.H. Shen, J.M. Shieh, Y.L. Chueh, Enhanced solar performance of chemical bath deposited$\mathrm{Zn}(0, \mathrm{~S}) / \mathrm{Cu}(\mathrm{In}, \mathrm{Ga}) \mathrm{Se}_{2}$ solar cells via interface engineering by a wet soaking process, J. Mater. Chem. A, 3 (2015) 14985-14990.

[7] J.F. Guillemoles, L. Kronik, D. Cahen, U. Rau, A. Jasenek, H.W. Schock, Stability issues of $\mathrm{Cu}(\mathrm{In}, \mathrm{Ga}) \mathrm{Se}_{2}$-based solar cells, J. Phys. Chem. B, 104 (2000) 4849-4862.

[8] F.Q. Huang, C.Y. Yang, D.Y. Wan, Advanced solar materials for thin-film photovoltaic cells, Front. Phys., 6 (2011) 177-196.

[9] T. Todorov, D.B. Mitzi, Direct Liquid Coating of Chalcopyrite Light-Absorbing Layers for Photovoltaic Devices, Eur. J. Inorg. Chem., (2010) 17-28.

[10] A. Eicke, T. Ciba, D. Hariskos, R. Menner, C. Tschamber, W. Witte, Depth profiling with SNMS and SIMS of $\mathrm{Zn}(\mathrm{O}, \mathrm{S})$ buffer layers for $\mathrm{Cu}(\mathrm{In}, \mathrm{Ga}) \mathrm{Se}_{2}$ thin-film solar cells, Surf. Interface Anal., 45 (2013) 1811-1820.

[11] C. Schwartz, D. Nordlund, T.-C. Weng, D. Sokaras, L. Mansfield, A.S. Krishnapriyan, K. Ramanathan, K.E. Hurst, D. Prendergast, S.T. Christensen, Electronic structure study of the CdS buffer layer in CIGS solar cells by X-ray absorption spectroscopy: Experiment and theory, Solar Energy Materials and Solar Cells, 149 (2016) 275-283.

[12] A. Abdel-Galil, M.R. Balboul, A. Atta, I.S. Yahia, A. Sharaf, Preparation, structural and optical characterization of nanocrystalline CdS thin film, Physica B, 447 (2014) 35-41.

[13] M. Powalla, W. Witte, P. Jackson, S. Paetel, E. Lotter, R. Wuerz, F. Kessler, C. Tschamber, W. Hempel, D. Hariskos, R. Menner, A. Bauer, S. Spiering, E. Ahlswede, T.M. Friedlmeier, D. Blazquez-Sanchez, I. Klugius, W. Wischmann, CIGS Cells and Modules With High Efficiency on Glass and Flexible Substrates, IEEE J. Photovolt., 4 (2014) 440-446.

[14] J. Stöhr, NEXAFS Spectroscopy, Springer-Verlag, New York, 2003.

[15] D. Prendergast, G. Galli, X-ray absorption spectra of water from first principles calculations, Phys. Rev. Lett., 96 (2006) 215502.

[16] J.N. Alexander, S. Higashiya, D. Caskey, H. Efstathiadis, P. Haldar, Deposition and characterization of cadmium sulfide (CdS) by chemical bath deposition using an alternative chemistry cadmium precursor, Solar Energy Materials and Solar Cells, 125 (2014) 47-53. 
[17] M. Bar, I. Repins, M.A. Contreras, L. Weinhardt, R. Noufi, C. Heske, Chemical and electronic surface structure of 20\%-efficient $\mathrm{Cu}(\mathrm{In}, \mathrm{Ga}) \mathrm{Se}_{2}$ thin film solar cell absorbers, Appl. Phys. Lett., 95 (2009) 052106.

[18] D. Eich, U. Herber, U. Groh, U. Stahl, C. Heske, M. Marsi, M. Kiskinova, W. Riedl, R. Fink, E. Umbach, Lateral inhomogeneities of $\mathrm{Cu}(\mathrm{In}, \mathrm{Ga}) \mathrm{Se}-2$ absorber films, Thin Solid Films, 361 (2000) 258-262.

[19] C. Heske, D. Eich, R. Fink, E. Umbach, T. van Buuren, C. Bostedt, S. Kakar, L.J.

Terminello, M.M. Grush, T.A. Callcott, F.J. Himpsel, D.L. Ederer, R.C.C. Perera, W. Riedl, F. Karg, Semi-quantitative and non-destructive analysis of impurities at a buried interface: Na and the $\mathrm{CdS} / \mathrm{Cu}(\mathrm{In}, \mathrm{Ga}$ )Se 2 heterojunction, Surf. Interface Anal., 30 (2000) 459-463. [20] C. Heske, U. Groh, L. Weinhardt, O. Fuchs, B. Holder, E. Umbach, C. Bostedt, L.J. Terminello, S. Zweigart, T.P. Niesen, F. Karg, Damp-heat induced sulfate formation in $\mathrm{Cu}(\mathrm{In}, \mathrm{Ga})(\mathrm{S}, \mathrm{Se})_{2}$-based thin film solar cells, Appl. Phys. Lett., 81 (2002) 4550-4552.

[21] L. Weinhardt, M. Bar, S. Pookpanratana, M. Morkel, T.P. Niesen, F. Karg, K. Ramanathan, M.A. Contreras, R. Noufi, E. Umbach, C. Heske, Sulfur gradient-driven Se diffusion at the CdS/CuIn(S,Se)2 solar cell interface, Appl. Phys. Lett., 96 (2010) 182102.

[22] W.J. Li, S.R. Cohen, D. Cahen, Effect of chemical treatments on nm-scale electrical characteristics of polycrystalline thin film $\mathrm{Cu}(\mathrm{In}, \mathrm{Ga}) \mathrm{Se}_{2}$ surfaces, Solar Energy Materials and Solar Cells, 120 (2014) 500-505.

[23] M.A. Contreras, M.J. Romero, B.T.E. Hasoon, R. Noufi, S. Ward, K. Ramanathan, Optimization of CBD CdS process in high-efficiency $\mathrm{Cu}(\mathrm{In}, \mathrm{Ga}) \mathrm{Se}_{2}$-based solar cells, Thin Solid Films, 403 (2002) 204-211.

[24] A.H. England, A.M. Duffin, C.P. Schwartz, J.S. Uejio, D. Prendergast, R.J. Saykally, On the hydration and hydrolysis of carbon dioxide, Chem. Phys. Lett., 514 (2011) 187-195.

[25] J.P. Perdew, K. Burke, M. Ernzerhof, Generalized gradient approximation made simple, Phys. Rev. Lett., 77 (1996) 3865-3868.

[26] R. Peverati, D.G. Truhlar, Quest for a universal density functional: the accuracy of density functionals across a broad spectrum of databases in chemistry and physics, Philos. Trans. R. Soc. A-Math. Phys. Eng. Sci., 372 (2014) 191102.

[27] P. Giannozzi, S. Baroni, N. Bonini, M. Calandra, R. Car, C. Cavazzoni, D. Ceresoli, G.L. Chiarotti, M. Cococcioni, I. Dabo, A. Dal Corso, S. de Gironcoli, S. Fabris, G. Fratesi, R. Gebauer, U. Gerstmann, C. Gougoussis, A. Kokalj, M. Lazzeri, L. Martin-Samos, N. Marzari, F. Mauri, R. Mazzarello, S. Paolini, A. Pasquarello, L. Paulatto, C. Sbraccia, S. Scandolo, G. Sclauzero, A.P. Seitsonen, A. Smogunov, P. Umari, R.M. Wentzcovitch, QUANTUM ESPRESSO: a modular and open-source software project for quantum simulations of materials, J. Phys.Condes. Matter, 21 (2009) 395502.

[28] G. Bergerhoff, R. Hundt, R. Sievers, I.D. Brown, THE INORGANIC CRYSTAL-STRUCTURE DATA-BASE, J. Chem. Inf. Comput. Sci., 23 (1983) 66-69.

[29] I. Ishii, A.P. Hitchcock, The Oscillator-Strengths for C1s and 01s Excitation of Some Saturated and Unsaturated Organic Alcohols, Acids and Esters, J Electron Spectrosc, 46 (1988) 55-84.

[30] D.A. Outka, J. Stohr, R.J. Madix, H.H. Rotermund, B. Hermsmeier, J. Solomon, Nexafs Studies of Complex Alcohols and Carboxylic-Acids on the Si(111)(7x7) Surface, Surf Sci, 185 (1987) 53-74. 
[31] S.G. Urquhart, A.P. Hitchcock, R.D. Priester, E.G. Rightor, Analysis of Polyurethanes Using Core Excitation Spectroscopy .2. Inner-Shell Spectra of Ether, Urea and Carbamate Model Compounds, J Polym Sci Pol Phys, 33 (1995) 1603-1620.

[32] M. Grioni, J.F. van Acker, M.T. Czyzyk, J.C. Fuggle, Unoccupied electronic structure and core-hole effects in the X-ray absorption spectra of $\mathrm{Cu}_{2} \mathrm{O}$, Physical Review B, 45 (1992) 3309-3318.

[33] T. Pylkkanen, J. Lehtola, M. Hakala, A. Sakko, C. Monaco, S. Huotari, K. Hamalainen, Universal Signature of Hydrogen Bonding in the Oxygen K-Edge Spectrum of Alcohols, J. Phys. Chem. B, 114 (2010) 13076-13083.

[34] A.B. Gurevich, B.E. Bent, A.V. Teplyakov, J.G. Chen, A NEXAFS investigation of the formation and decomposition of $\mathrm{CuO}$ and $\mathrm{Cu}_{2} \mathrm{O}$ thin films on $\mathrm{Cu}(100)$, Surf Sci, 442 (1999) L971-L976.

[35] J.A. Sellberg, S. Kaya, V.H. Segtnan, C. Chen, T. Tyliszczak, H. Ogasawara, D. Nordlund, L.G.M. Pettersson, A. Nilsson, Comparison of x-ray absorption spectra between water and ice: New ice data with low pre-edge absorption cross-section, The Journal of chemical physics, 141 (2014) 034507.

[36] C. Haiyan, W. Wen, W. Qi, J.C. Hanson, J.T. Muckerman, E. Fujita, A.I. Frenkel, J.A. Rodriguez, Preparation of $\left(\mathrm{Ga}_{1-\mathrm{x}} \mathrm{Zn}_{\mathrm{x}}\right)\left(\mathrm{N}_{1-\mathrm{x}} \mathrm{O}_{\mathrm{x}}\right)$ photocatalysts from the reaction of $\mathrm{NH}_{3}$ with $\mathrm{Ga}_{2} \mathrm{O}_{3} \mathrm{ZnO}$ and $\mathrm{ZnGa}_{2} \mathrm{O}_{4}$ : in situ time-resolved XRD and XAFS studies, J. Phys. Chem. C, 113 (2009) 3650-3659.

[37] C. McGuinness, C.B. Stagarescu, P.J. Ryan, J.E. Downes, D.F. Fu, K.E. Smith, R.G. Egdell, Influence of shallow core-level hybridization on the electronic structure of post-transitionmetal oxides studied using soft X-ray emission and absorption, Physical Review B, 68 (2003) 165104.

[38] J. Chen, C.L. Lin, S.L. Qiu, M. Strongin, M.L. Denboer, Auger and X-ray absorption studies of solid molecular-oxygen, J. Vac. Sci. Technol. A-Vac. Surf. Films, 8 (1990) 2591-2594. [39] K. Ramanathan, F.S. Hasoon, S. Smith, D.L. Young, M.A. Contreras, P.K. Johnson, A.O. Pudov, J.R. Sites, Surface treatment of CuInGaSe 2 thin films and its effect on the photovoltaic properties of solar cells, Journal of Physics and Chemistry of Solids, 64 (2003) 1495-1498. [40] G. Vanderlaan, R.A.D. Pattrick, C.M.B. Henderson, D.J. Vaughan, Oxidation-state variations in copper minerals sudied with $\mathrm{Cu} 2 \mathrm{p}$ X-ray absorption spectroscopy, Journal of Physics and Chemistry of Solids, 53 (1992) 1185-1190.

[41] E. Kosobrodova, A. Kondyurin, W. Chrzanowski, D.G. McCulloch, D.R. McKenzie, M.M.M. Bilek, Optical properties and oxidation of carbonized and cross-linked structures formed in polycarbonate by plasma immersion ion implantation, Nucl. Instrum. Methods Phys. Res. Sect. B-Beam Interact. Mater. Atoms, 329 (2014) 52-63. 